\title{
Pien Tze Huang induces apoptosis in multidrug-resistant U2OS/ADM cells via downregulation of Bcl-2, survivin and P-gp and upregulation of Bax
}

\author{
YAN ZHANG ${ }^{1}$, QIHONG WANG ${ }^{2}$, SUSHENG NIU $^{1}$, JUNNING LIU $^{1}$ and LI ZHANG ${ }^{1}$ \\ ${ }^{1}$ College of Osteopedics and Traumatology, Fujian University of Traditional Chinese Medicine, Fuzhou, Fujian 350122; \\ ${ }^{2}$ First Affiliated People's Hospital of Fujian University of Traditional Chinese Medicine, Fuzhou, Fujian 350004, P.R. China
}

Received October 5, 2013; Accepted November 26, 2013

DOI: $10.3892 /$ or.2013.2916

\begin{abstract}
Pien Tze Huang (PZH) is a well-known traditional Chinese formula that was first prescribed by a royal physician in the Ming Dynasty. PZH has been used to treat various types of cancers including osteosarcoma. Previous studies have shown that PZH may effectively inhibit osteosarcoma cell growth in vivo and in vitro via induction of apoptosis and inhibition of migratory and invasive abilities. However, little is known regarding the effects of PZH on osteosarcomas that are resistant to chemotherapy, which has emerged as a major clinical problem. In the present study, the cellular effects of PZH on multidrug-resistant U2OS/ADM human osteosarcoma cells were investigated. Our results showed that $\mathrm{PZH}$ reduced cell viability in a dose- and time-dependent manner and arrested cells in the $\mathrm{G}_{2} / \mathrm{M}$ phase of the cell cycle, suggesting that PZH inhibits the proliferation of U2OS/ADM cells. Hoechst 33258 staining and Annexin V/propidium iodide double staining revealed typical nuclear features of apoptosis, and treatment with PZH increased the proportion of apoptotic Annexin V-positive cells in a dose-dependent manner. Further experiments demonstrated that apoptosis induction by $\mathrm{PZH}$ was accompanied by downregulation of Bcl-2 and survivin and upregulation of Bax. In addition, following treatment with PZH, intracellular Rhodamine 123 accumulation was increased and the expression of P-gp was significantly
\end{abstract}

Correspondence to: Dr Li Zhang, College of Osteopedics and Traumatology, Fujian University of Traditional Chinese Medicine, 1 Huatuo Road, Shangjie Minhou, Fuzhou, Fujian 350122, P.R. China

E-mail: zhangli1626@163.com

Abbreviations: PZH, Pien Tze Huang; OS, osteosarcoma; P-gp, P-glycoprotein; TCM, Traditional Chinese medicine; IAP, inhibitor of apoptosis; DMEM, Dulbecco's modified Eagle's medium; FBS, fetal bovine serum; ADM, adriamycin; $\mathrm{IC}_{50}, 50 \%$ inhibitory concentration

Key words: Pien Tze Huang, osteosarcoma, multidrug-resistance, apoptosis suppressed. Taken together, these results provide a possible molecular mechanism for the anticancer effect of PZH on U2OS/ADM cells and suggest that PZH may be a potent therapeutic agent for drug-resistant osteosarcoma.

\section{Introduction}

Osteosarcoma (OS) is the most common primary bone malignancy mainly affecting children and adolescents with an extremely high propensity for local invasion and distant metastasis $(1,2)$. Chemotherapy has become a cornerstone for the primary treatment of osteosarcoma. Multimodal treatment regimens usually involve neoadjuvant chemotherapy with highdose methotrexate, doxorubicin, cisplain and more recently ifosfamide. This regimen has improved patient survival from $20 \%$ with surgical resection alone to $70 \%$ for localized disease (3). However, despite intensive efforts in both surgical and medical management, the survival rate has not improved over the last 30 years, and $40 \%$ of OS patients succumb to the disease (4). Multidrug resistance (MDR) is a formidable barrier to the success of cancer chemotherapy $(5,6)$. Therefore, researchers are working intensely to discover new anticancer drugs as therapeutic regimens against OS.

Natural products, including plants, animals and microorganisms, have played a major role in new drug discovery for centuries, with over $74.9 \%$ of approved anticancer agents being of natural origin $(7,8)$. Traditional Chinese medicine (TCM) uses natural products guided by TCM theories, to treat various diseases. TCM has been confirmed to possess effective anticancer drugs against cancers including OS (9-11), and even against drug-resistant cells (12-14). Pien Tze Huang $(\mathrm{PZH})$ is a well-known traditional Chinese formula that was first prescribed 450 years ago in the Ming Dynasty. The main ingredients of PZH include Moschus, Calculus bovis, snake gall and Radix Notoginseng. The main efficacy of $\mathrm{PZH}$ is heat-clearing, detoxification, promotion of blood circulation, reduction in blood stasis and swelling (15). According to TCM theories, the pathogenesis of cancer is related to accumulation of toxic dampness and heat and stagnation of blood stasis, thus PZH has been used to treat various types of cancers (16-19). Our previous studies revealed that PZH is able to inhibit OS growth in vivo and in vitro via induction of apoptosis and inhi- 
bition of migratory and invasive abilities (20-23). However, little is known regarding the effects of $\mathrm{PZH}$ on chemotherapyresistant OS cell lines.

OS cells employ a host of different mechanisms against resistant to one or more chemotherapeutic drugs. Abnormal expression of apoptosis-related proteins is closely related to chemotherapeutic drug resistance. Previous reports indicate that Bcl-2 family proteins are expressed at a high level in OS (24). Apoptosis is regulated by the balance of pro- and anti-apoptotic members of the Bcl-2 family proteins. Antiapoptotic Bcl-2 protein is a key protein that blocks apoptosis; overexpression of anti-apoptotic Bcl-2 protein is closely related to evasion of apoptosis and increased chemotherapy resistance. In contrast, pro-apoptotic Bax proteins can improve the sensitivity of malignant cells to apoptosis, thereby overcoming drug resistance $(25,26)$. Survivin protein which belongs to the inhibitor of apoptosis (IAP) family has two known functions: regulation of cell division and inhibition of apoptosis. It has been widely demonstrated that overexpression of survivin causes resistance to various chemotherapeutic agents (27). P-glycoprotein (P-gp), a transmembrane glycoprotein, that functions as a drug efflux pump, reduces the intercellular accumulation and toxicity of numerous anticancer drugs, including doxorubicin, paclitaxel, and the vinca alkaloids. The overexpression of P-gp is one of the most studied mechanisms of drug resistance $(28,29)$. In addition, P-gp also plays a special role in the caspase-dependent apoptosis pathway in drug-resistant cancer cells (30).

In order to extend the clinical observations of the potential anticancer effect of $\mathrm{PZH}$ and help to elucidate the mechanism of its anticancer activity, in the present study, the cellular effects of PZH on multidrug-resistant OS U2OS/ADM cells were investigated, and the changes in apoptosis and drug-resistance-related factors were also examined. We found that PZH significantly inhibited the growth of U2OS/ADM cells through arrest in the $\mathrm{G}_{2} / \mathrm{M}$ phase of the cell cycle and promoted apoptosis of U2OS/ADM cells by downregulation of the expression of $\mathrm{Bcl}-2$ and survivin and upregulation of the expression of Bax; at the same time $\mathrm{P}$-gp expression was inhibited. These data indicate that PZH is a valuable agent that may be useful for treating OS patients with drug resistance.

\section{Materials and methods}

Materials and reagents. Dulbecco's modified Eagle's medium (DMEM), fetal bovine serum (FBS), Hoechst 33258, TRIzol reagent, penicillin-streptomycin were obtained from Invitrogen Inc. (Grand Island, NY, USA). Trypsin was purchased from HyClone Laboratories Inc. (Logan, UT, USA). Rhodamine was purchased from Sigma (St. Louis, MO, USA). Cycle Test Plus DNA Reagent kit and an apoptosis assay (FITC Annexin V-FITC Apoptosis Detection Kit II) were provided by Becton-Dickinson (San Jose, CA, USA). The Bcl-2, Bax, survivin and GAPDH primers were purchased from Sangon Biotech Co., Ltd. (Shanghai, China). Bcl-2, Bax, survivin and P-gp antibodies, horseradish peroxidase (HRP)-conjugated secondary antibodies and the antibody against $\beta$-actin were obtained from Cell Signaling Technology, Inc. (Danvers, MA, USA).
Preparation of $\mathrm{PZH}$. PZH was obtained from and authenticated by the sole manufacturer Zhangzhou Pien Tze Huang Pharmaceutical Co., Ltd. China (Chinese FDA approval no: Z35020242). Stock solutions were prepared by dissolving PZH power in $10 \%$ dimethyl sulfoxide (DMSO) to a concentration of $30 \mathrm{mg} / \mathrm{ml}$. The working concentrations of $\mathrm{PZH}$ were obtained by diluting the stock solution in the culture medium. The final concentrations of DMSO in the medium were $<1 \%$.

Cell lines and cell culture. The human OS cell line U2OS was obtained from the Institute of Biochemistry and Cell Biology, Chinese Academy of Sciences (Shanghai, China). The multidrug-resistant OS cell line U2OS/ADM, which overexpresses multidrug resistance protein 1 (MDR1, also known as P-gp) and multidrug resistance-associated protein (MRP1), was selected in a step-wise manner by exposing drug-sensitive parental cells to increasing doses of adriamycin (ADM) (31). Both cell lines were grown as adherent monolayers in a flask with DMEM culture medium containing $10 \% \mathrm{FBS}$ and $100 \mathrm{U} /$ $\mathrm{ml}$ penicillin and $100 \mu \mathrm{g} / \mathrm{ml}$ streptomycin at $37^{\circ} \mathrm{C}$ in a humidified atmosphere of $5 \% \mathrm{CO}_{2}$. To maintain drug resistance, $1 \mu \mathrm{g} /$ ml ADM was supplemented at a regular interval of 2 days, but was omitted 2 weeks before any of the experiments. Logarithmically growing cells were used for all experiments.

Cell viability studies. The effects of $\mathrm{PZH}$ on the proliferation of U2OS/ADM and U2OS cells were measured by MTT colorimetric assay. Cells were seeded at $1 \times 10^{4}$ cells/well in 96-well plates (Corning Costar Corporation, Corning, NY, USA). After $24 \mathrm{~h}$ of incubation with fresh medium, various concentrations of PZH were added to the plates. The number of viable cells was determined at daily intervals $(24,48$ and $72 \mathrm{~h})$. At the end of the treatment, $100 \mu \mathrm{l}$ MTT [0.5 mg/ml in phosphate-buffered saline (PBS)] was added to each well, and the samples were incubated for an additional $4 \mathrm{~h}$ at $37^{\circ} \mathrm{C}$. The purple-blue MTT formazan precipitate was dissolved in $100 \mu \mathrm{l}$ DMSO. The absorbance was measured at $570 \mathrm{~nm}$ using an ELx808 ${ }^{\mathrm{TM}}$ absorbance microplate reader (BioTek Instruments Inc., Winooski, VT, USA). The relative cell viability was expressed as the ratio (\%) of the absorbance in the experimental wells to that of the control wells (normal culture medium with $1 \%$ DMSO). The $50 \%$ inhibitory concentration $\left(\mathrm{IC}_{50}\right)$ was calculated.

Cell cycle assays. Cell cycle analysis was carried out by a flow cytometry assay and the Cycle Test Plus DNA Reagent kit. Briefly, U2OS/ADM cells were treated with PZH for $48 \mathrm{~h}$ at concentrations of $0.4,0.8$ and $1.6 \mathrm{mg} / \mathrm{ml}$. Control cells were treated with normal culture medium with $1 \%$ DMSO. After incubation, cells were harvested by trypsinization and washed twice with ice cold PBS. PI staining was performed according to the Cycle Test Plus DNA Reagent kit manufacturer's recommendations. Finally, the cell cycle distribution was determined using fluorescence-activated cell sorting (FACSCalibur; Becton-Dickinson, San Jose, CA, USA). The proportion of DNA in different phases was analyzed using ModFit LT version 3.0 (Verity Software House, Topsham, ME, USA).

Hoechst 33258 staining assay. U2OS/ADM cells were seeded into 6 -well plates at a density of $2.0 \times 10^{5}$ cells/well and incubated for $24 \mathrm{~h}$ to allow cell attachment. Different concen- 
trations of $\mathrm{PZH}$ were then added to each well and incubated for an additional $48 \mathrm{~h}$, and the cells were then washed with ice cold PBS twice and fixed with $4 \%$ paraformaldehyde for $15 \mathrm{~min}$ at room temperature. The cells were incubated in $1 \mathrm{ml}$ PBS containing $10 \mu \mathrm{mol} / 1$ Hoechst 33258 at $37^{\circ} \mathrm{C}$ for $30 \mathrm{~min}$. Fluorescence microscopy (Olympus, Japan) was used to observe the apoptotic characteristics of nuclear condensation.

Annexin V/propidium iodide staining analysis by flow cytometry. Following incubation with various doses of $\mathrm{PZH}$ for $48 \mathrm{~h}$, apoptosis of U2OS/ADM cells was determined by flow cytometric (FCM) analysis using fluorescence-activated cell sorting (FACSCalibur; Becton-Dickinson) and the Annexin V-FITC Apoptosis Detection Kit II. Staining was performed according to the manufacturer's instructions. The percentage of cells in early apoptosis was calculated by Annexin V-positivity and PI-negativity, while the percentage of cells in late apoptosis was calculated by Annexin V-positivity and PI-positivity.

Rhodamine 123 accumulation assay. Rhodamine 123 was used to evaluate the transport function of P-gp in U2OS/ADM cells by flow cytometric analysis. Cells $\left(2.0 \times 10^{5}\right.$ cells/well in 6 -well plates) were treated with different concentrations of $\mathrm{PZH}$ for $48 \mathrm{~h}$, followed by addition of Rhodamine $123(5 \mu \mathrm{g} / \mathrm{ml})$. After incubating at $37^{\circ} \mathrm{C}$ for $30 \mathrm{~min}$, the cells were harvested and washed twice with ice-cold PBS and subsequently analyzed by flow cytometry. The values are expressed as the mean fluorescence intensity of Rhodamine 123.

RNA extraction and RT-PCR analysis. U2OS/ADM cells were seeded into $25-\mathrm{cm}^{2}$ culture flasks at a density of $1 \times 10^{5}$ cells $/ \mathrm{ml}$ in $4 \mathrm{ml}$ of medium and treated with various doses of PZH for $48 \mathrm{~h}$. Total RNA from U2OS/ADM cells was isolated with TRIzol reagent (Invitrogen). Oligo(dT)-primed RNA $(1 \mu \mathrm{g})$ was reverse-transcribed with SuperScript II reverse transcriptase (Promega, Madison, WI, USA) according to the manufacturer's instructions. The obtained cDNA was used to determine the mRNA amount of Bcl-2, Bax and survivin by PCR with Taq DNA polymerase (Fermentas). GAPDH was used as an internal control. The primers and the annealing temperature $\left({ }^{\circ} \mathrm{C}\right)$ used for amplification of Bcl-2, Bax, survivin and GAPDH transcripts are as follows: Bcl-2 forward, 5'-CAG CTG CAC CTG ACG CCC TT-3 and reverse, 5'-GCC TCC GTT ATC CTG GAT CC-3', 55 C; Bax forward, 5'-TGC TTC AGG GTT TCA TCC AGG-3' and reverse, 5'-TGG CAA AGT AGA AAA GGG CGA-3', 55 ${ }^{\circ}$; survivin forward, 5'-ACC ACC GCA TCT CTA CAT TC-3' and reverse, 5'-GTT CCT CTA TGG GGT CGT C-3', 55 ${ }^{\circ}$; P-gp forward, 5'-TAG AAA ACT TCC GAA CCG TTG T-3' and reverse, 5'-TAG CTG TCA ATC AAA GGG GTT T-3', 55 C; GAPDH forward, 5'-AGA AGG CTG GGG CTC ATT TG-3' and reverse, 5'-AGG GGC CAT CCA CAG TCT TC-3', 55 $\mathrm{C}$. PCR products were visualized on a $1.5 \%$ agarose gel. The DNA bands were examined using a Gel Documentation System (Model Gel Doc 2000; Bio-Rad Laboratories, Hercules, CA, USA). Intensities of the mRNA levels were normalized to those of the GAPDH products as ratios to produce arbitrary units of relative abundance.

Western blot analysis. U2OS/ADM cells were treated with various doses of $\mathrm{PZH}$ for $48 \mathrm{~h}$. The cells were harvested;
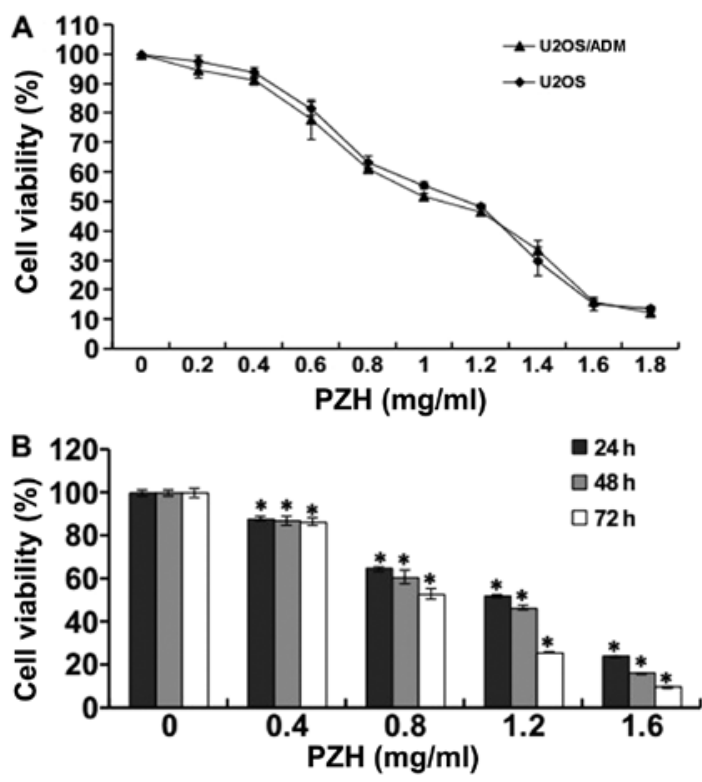

Figure 1. Anti-proliferative effect of PZH on U2OS/ADM cells. (A) U2OS/ADM and U2OS cells were treated with PZH at the indicated concentrations for $48 \mathrm{~h}$. (B) U2OS/ADM cells were treated with the indicated concentrations of PZH for 24, 48 and $72 \mathrm{~h}$. Cell viability was measured by MTT assay. The data were normalized to the viability of the control cells Data are expressed as means \pm SD (error bars) of at least 3 independent experiments. " $\mathrm{P}<0.05$ vs. control cells. $\mathrm{PZH}$, Pien Tze Huang.

protein lysates from the cells were generated through the mammalian cell lysis buffer containing protease and phosphatase inhibitor cocktails. The quantification of the protein content was performed with the bicinchoninic acid (BCA) protein assay kit. Equal aliquots of protein lysate were separated by $12 \%$ SDS-PAGE, followed by electrophoresis and transferred to polyvinylidence difluoride (PVDF) membranes. Membranes were blocked for $1 \mathrm{~h}$ in $5 \%$ nonfat dry milk in TBS with Tween-20 (TBST) and probed overnight with appropriate dilutions of primary antibodies against Bcl-2 (1:1,000), Bax (1:500), survivin $(1: 1,000), \mathrm{P}-\mathrm{gp}(1: 1,000)$ and $\beta$-actin $(1: 1,000)$ at $4^{\circ} \mathrm{C}$. Three consecutive washes were performed for $10 \mathrm{~min}$ with TBST; the membranes were incubated with the secondary HRP-conjugated antibodies at a dilution of 1:2,000 for $1 \mathrm{~h}$ at room temperature. Finally, the membranes were washed again in TBST. Antibody-bound protein band detection was performed with the ECL Detection VersaDoc ${ }^{\mathrm{TM}}$ Imaging System (Bio-Rad Laboratories).

Statistical analysis. All of the data were confirmed by at least 3 independent experiments. Statistical analysis of data was carried out using the statistical software SPSS 13.0. Data are expressed in terms of means \pm SD. The statistical analysis of the results was performed by Student's t-test for paired samples. Differences between concentrations were analyzed statistically with ANOVA. A P-value $<0.05$ was considered to indicate a statistically significant result.

\section{Results}

PZH inhibits U2OS/ADM cell proliferation. To investigate the effects of PZH on the viability of U2OS/ADM cells, an MTT 

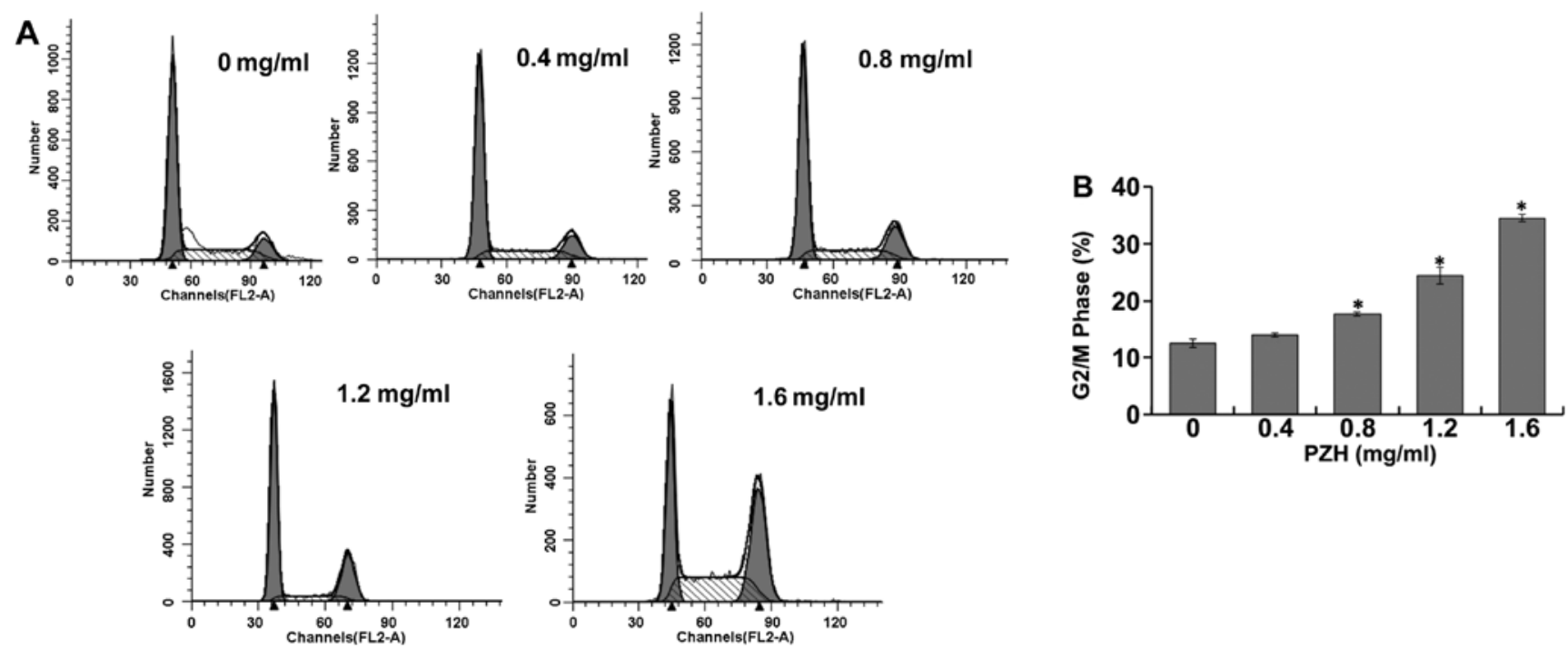

Figure 2. Effect of PZH on the cell cycle distribution of U2OS/ADM cells. (A) Following treatment with the indicated concentrations of PZH for $48 \mathrm{~h}$, U2OS/ ADM cells were collected and stained with PI followed by FACS analysis. (B) The proportion of DNA in the $\mathrm{G}_{2} / \mathrm{M}^{\mathrm{M}}$ phase is shown after treatment with the indicated concentrations of PZH for 48 h. Data are expressed as means \pm SD (error bars) of at least 3 independent experiments. " $\mathrm{P}<0.05 \mathrm{vs.} \mathrm{control} \mathrm{cells.} \mathrm{PZH,}$ Pien Tze Huang.

assay was performed. As shown in Fig. 1A, U2OS/ADM cells and their parental U2OS cells were exposed to different PZH concentrations for $48 \mathrm{~h}$, and PZH induced cell death in a dosedependent manner. The half-inhibitory concentration $\left(\mathrm{IC}_{50}\right)$ of $\mathrm{PZH}$ at $48 \mathrm{~h}$ in U2OS/ADM and U2OS cells was $\sim 1.06$ and $1.14 \mathrm{mg} / \mathrm{ml}$, respectively, suggesting that $\mathrm{PZH}$ has similar inhibitory effects on cell proliferation in both resistant and parental OS cells. Thus, drug-resistant OS cells are sensitive to PZH. Fig. 1B shows that the treatment of U2OS/ADM cells with PZH resulted in a significant inhibition of cell growth in a time-dependent manner $(\mathrm{P}<0.05$, vs. control group).

$P Z H$ induces U2OS/ADM cell cycle arrest at the $G_{2} / M$ phase. The cell cycle is a crucial regulator of cell proliferation. When the cell cycle is disturbed, the rate of cell proliferation is reduced or apoptosis is induced. We aimed to determine whether PZH causes cell cycle arrest. Cell cycle distribution was evaluated by flow cytometry after U2OS/ADM cells were exposed to $\mathrm{PZH}$ at various concentrations for $48 \mathrm{~h}$. As shown in Fig. 2A and B, the percentage of accumulated cells in the $\mathrm{G}_{2} / \mathrm{M}$ phase increased from $12.6 \%$ in the control group to $14.03,17.27,23.45$ and $35.11 \%$ in cells treated with $0.4,0.8$, 1.2 and $1.6 \mathrm{mg} / \mathrm{ml}$ of PZH for $48 \mathrm{~h}$, respectively. These results indicate that the inhibitory effect of PZH on U2OS/ADM cell proliferation was associated with $\mathrm{G}_{2} / \mathrm{M}$ phase cell cycle arrest.

PZH induces apoptosis in U2OS/ADM cells. To determine whether the inhibition of cell growth by PZH resulted from the induction of apoptosis, Hoechst 33258 staining was performed to observe changes in cell apoptosis induced by $\mathrm{PZH}$. Following treatment with different concentrations of PZH for $48 \mathrm{~h}$, the cells were analyzed by fluorescence microscopy. As shown in Fig. 3A, control cells showed round and homogeneous nuclei, while PZH-treated cells exhibited typical apoptotic morphologic changes including condensed and fragmented nuclei in a dose-dependent manner. To further study the apoptosis induced by $\mathrm{PZH}$, the cells undergoing apoptosis or necrosis were detected by FACS analysis after staining with Annexin V-FITC and PI. The percentage of apoptotic cells included both early apoptotic cells (lower right) and late apoptotic cells along with the necrotic fractions (upper right). As shown in Fig. 3B and C, the percentage of cells undergoing apoptosis following treatment with $0.4,0.8$, 1.2 and $1.6 \mathrm{mg} / \mathrm{ml}$ of PZH (including early and late apoptotic cells) was $8.32 \pm 0.93,20.28 \pm 1.39,35.54 \pm 1.65$ and $53.86 \pm 1.71 \%$, respectively ( $\mathrm{P}<0.05$, vs. control group). These results indicate that PZH treatment induces U2OS/ADM cell apoptosis in a dose-dependent manner.

PZH upregulates the expression of Bax and downregulates the expression of $\mathrm{Bcl}-2$ and survivin. It is well established that anti-apoptotic proteins Bcl-2 and survivin play an important role in preventing apoptosis in cancer cells while pro-apoptotic protein Bax has a reverse effect. To further study the mechanism of the induction of apoptosis by PZH activity, we performed RT-PCR and western blot analysis to examine the mRNA and protein expression of Bcl-2, survivin and Bax in PZH-treated U2OS/ADM cells. The results of the RT-PCR assay showed that the pro-apoptotic Bax was significantly upregulated and the anti-apoptotic Bcl-2 and survivin were significantly decreased; both effects occurred in a dose-dependent manner (Fig. 4A and B; $\mathrm{P}<0.05$, vs. control group), and the pattern of protein expression of Bax, Bcl-2 and survivin was similar to their respective mRNA levels (Fig. 4C). These results indicate that PZH induced apoptosis via the intrinsic pathway by upregulating expression of pro-apoptotic Bax and downregulating anti-apoptotic Bcl-2 and survivin in U2OS/ADM cells.

PZH decreases P-gp expression. Overexpression of P-gp, which reduces the intercellular accumulation and toxicity of many anticancer drugs, is one of the most studied mechanisms 
A

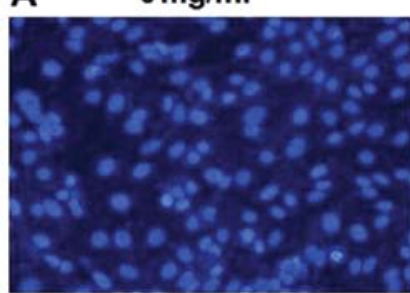

$0.4 \mathrm{mg} / \mathrm{ml}$

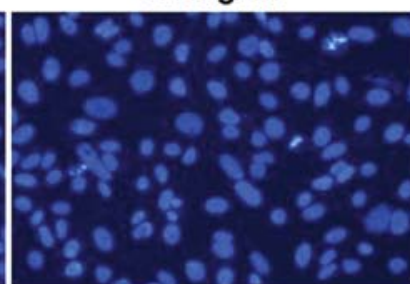

$0.8 \mathrm{mg} / \mathrm{ml}$

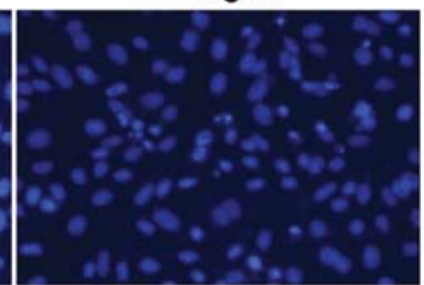

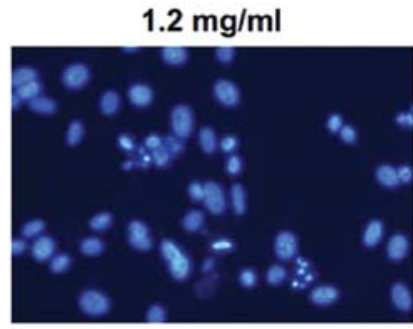

$1.6 \mathrm{mg} / \mathrm{ml}$
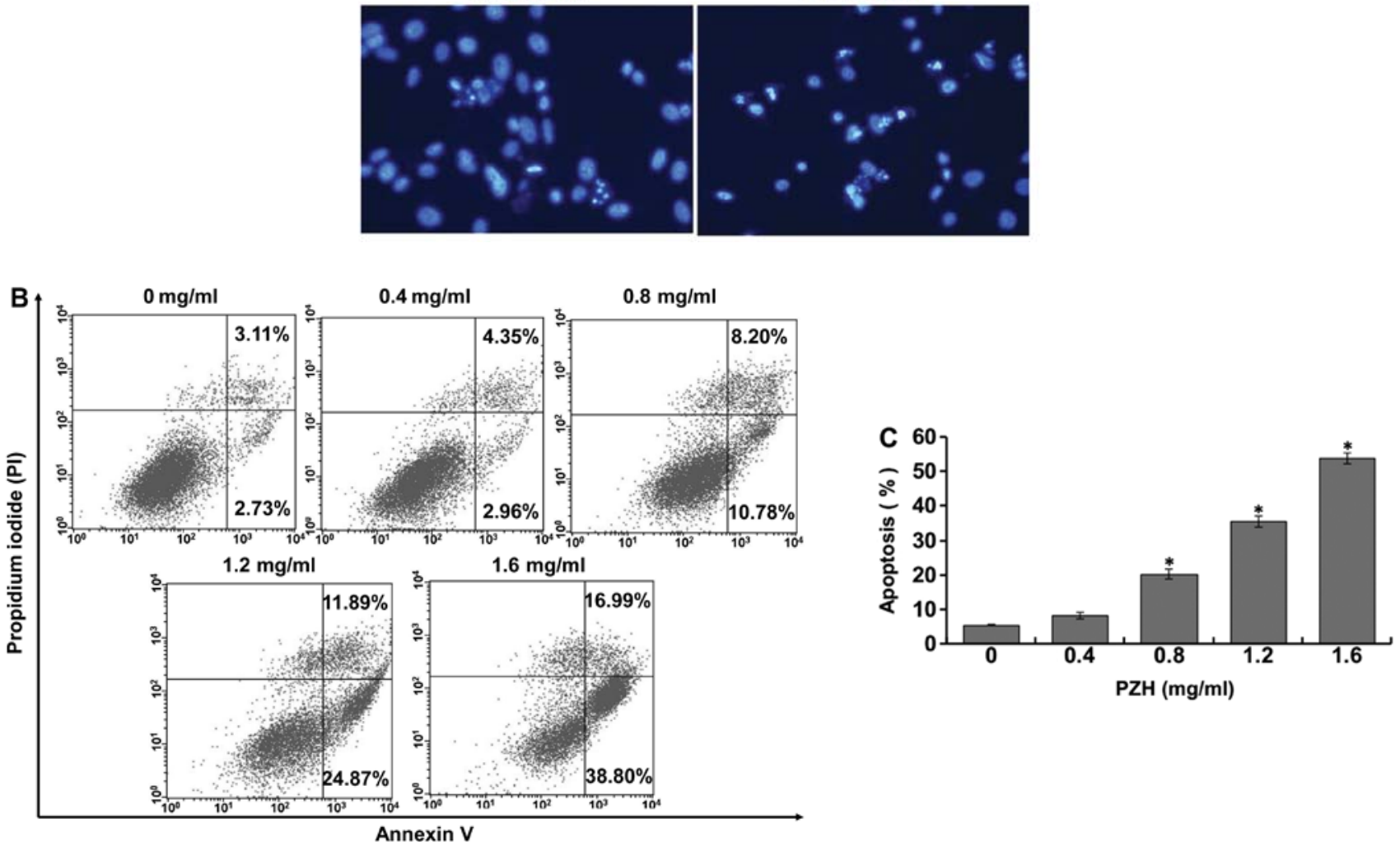

Figure 3. PZH induces apoptosis in U2OS/ADM cells. (A) Following treatment with the indicated concentrations of PZH for $48 \mathrm{~h}$, U2OS/ADM cells were stained with Hoechst 33258 and observed under a fluorescence microscope at $x 200$ magnification. PZH-treated cells exhibited typical apoptotic morphological changes including condensed and fragmented nuclei in a dose-dependent manner.(B) Induction of apoptosis by PZH in U2OS/ADM cells evaluated by Annexin V-FITC/PI staining. Quadrants represent the following populations: non-apoptotic viable cells in the lower left quadrant (Annexin V-negative/PI-negative); early apoptotic cells in the lower right quadrant (Annexin V-positve/PI-negative); late apoptotic or necrotic cells in the upper right quadrant (Annexin V-positve/PI-positve); and mechanically injured cells in the upper left quadrant (Annexin V-negative/PI-positive). (C) FACS results are expressed as means \pm SD (error bars). Three individual experiments were performed. ${ }^{*} \mathrm{P}<0.05$ vs. control cells. PZH, Pien Tze Huang.

of drug resistance. U2OS/ADM cells have been shown to express high levels of P-gp (31). To investigate whether PZH may modulate P-gp expression, the intracellular accumulation of Rhodamine 123 was examined by flow cytometry. As shown in Fig. 5A, the intracellular accumulation of Rhodamine 123 in U2OS/ADM cells was dose-dependently increased when compared to the control group after treatment with $\mathrm{PZH}(\mathrm{P}<0.05$, vs. control group). In addition, the expression of P-gp was also determined by RT-PCR and western blotting after treatment with various concentrations of $\mathrm{PZH}$. The results indicated that $\mathrm{PZH}$ treatment profoundly and dosedependently reduced the expression of P-gp (Fig. 5B and C).

\section{Discussion}

Drug resistance to chemotherapeutic agents is a major obstacle to the treatment of human osteosarcoma. Despite the numerous studies that have attempted to develop and discover effective chemotherapeutic drugs or reversal agents, the successful modulation of clinical drug resistance has not been achieved $(32,33)$. Thus, it is imperative to develop less toxic and more efficient therapeutic anticancer agents. TCM has recently been recognized as a new source of anticancer drugs and new chemotherapy adjuvants to enhance the efficacy of chemotherapy and to diminish side-effects and the resistance of cancer chemotherapies (34). PZH, a wellknown traditional Chinese formula which was first prescribed 450 years ago in the Ming Dynasty, has been used to treat various types of cancers (16-19). Our previous studies revealed that PZH significantly inhibited the proliferation of U2OS parental cells, arrested the cell cycle in the $\mathrm{G}_{2} / \mathrm{M}$ phase and promoted apoptosis $(20,22)$. However, the effects of PZH on chemotherapy-resistant osteosarcoma cells are still largely unknown. Therefore, in order for PZH to be further developed 

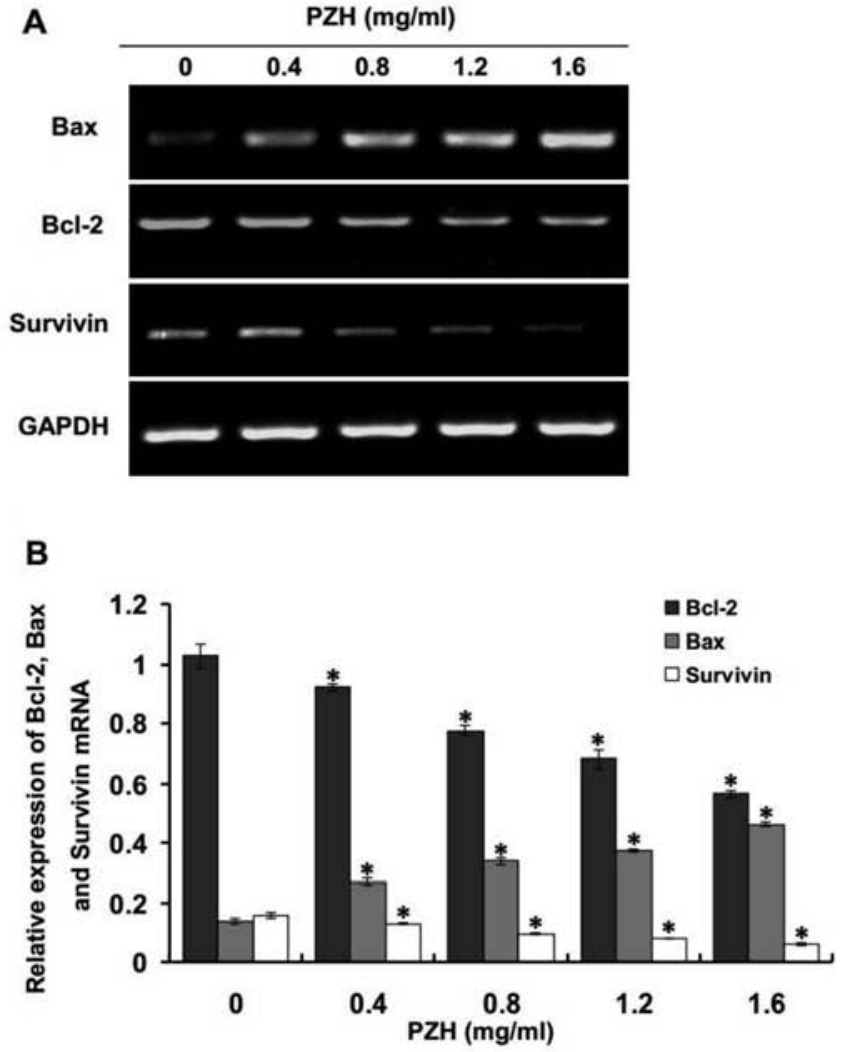

C

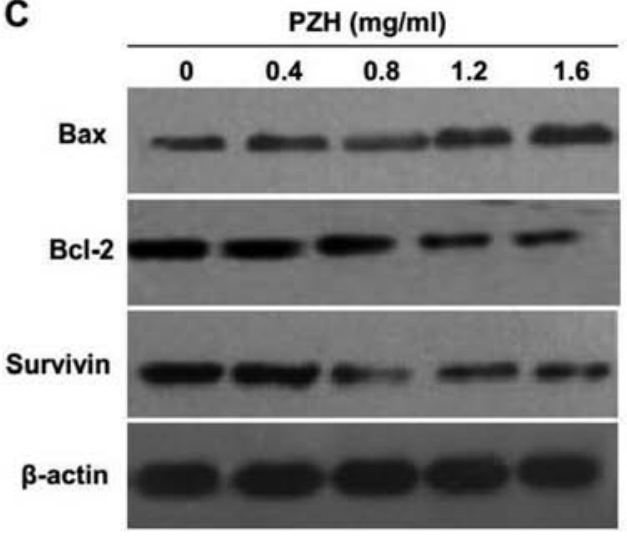

Figure 4. PZH upregulates the expression of Bax and downregulates the expression of Bcl-2 and survivin. The mRNA and protein expression of Bax, Bcl-2 and survivin was analyzed following exposure of U2OS/ADM cells to PZH at the indicated concentrations for $48 \mathrm{~h}$. (A) The mRNA levels of Bax, Bcl-2 and survivin were determined by RT-PCR. GAPDH was used as the internal control. (B) Relative signal intensities of mRNA are shown against GAPDH. " $\mathrm{P}<0.05$ vs. control cells. (C) Western blot analysis of protein expression of Bax, Bcl-2 and survivin in U2OS/ADM cells following treatment with PZH. $\beta$-actin was used as the internal control. Results are expressed as means $\pm \mathrm{SD}$ (error bars). Three individual experiments were performed. PZH, Pien Tze Huang.

as an anticancer agent, its inhibition of chemotherapy-resistant OS cell proliferation and the underlying mechanisms must be elucidated.

We first tested PZH for cytotoxicity against chemotherapy-resistant OS U2OS/ADM cells and the parental cells in vitro. We found that $\mathrm{PZH}$ had a marked inhibitory effect on the cell proliferation in both the resistant and sensitive OS
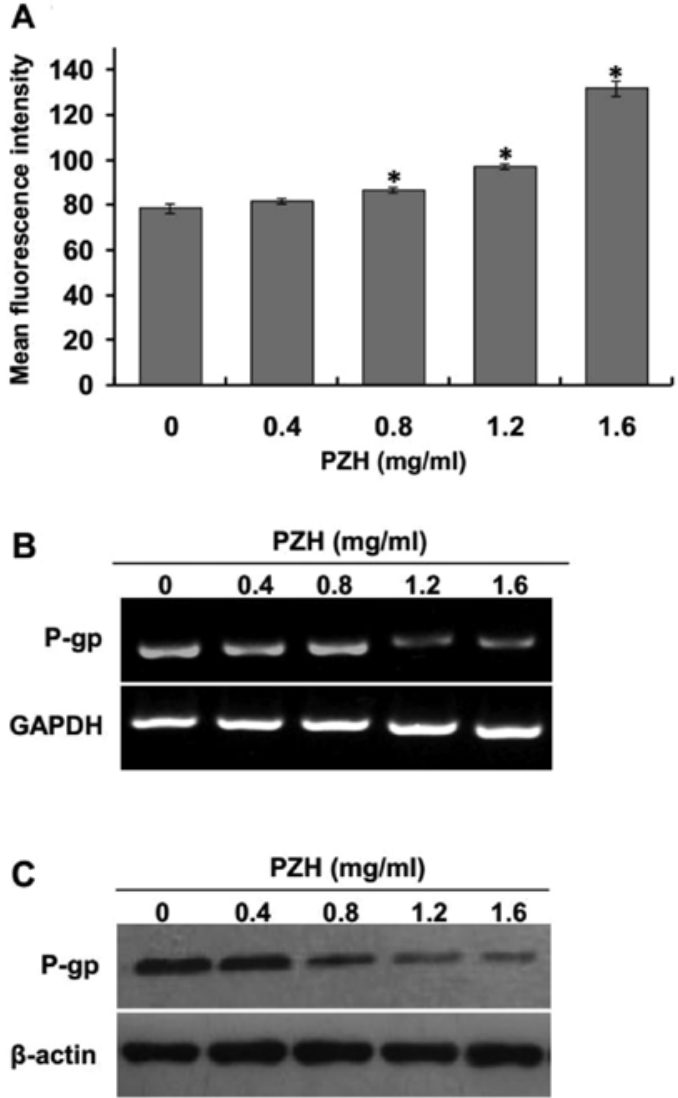

Figure 5. PZH increases the intracellular Rhodamine 123 accumulation and decreases P-gp expression. (A) Effect of PZH on the accumulation of Rhodamine 123 in U2OS/ADM cells. Following treatment with different concentrations of PZH for $48 \mathrm{~h}$, Rhodamine $123(5 \mu \mathrm{g} / \mathrm{ml})$ was added and incubation was carried out at $37^{\circ} \mathrm{C}$ for $30 \mathrm{~min}$ followed by flow cytometric analysis. The results are expressed as the mean fluorescence intensity of Rhodamine 123 . ${ }^{*} \mathrm{P}<0.05$ vs. control cells. (B) The mRNA levels of P-gp in U2OS/ADM cells treated with PZH were determined by RT-PCR. GAPDH was used as the internal control. (C) The protein expression of P-gp in U2OS/ ADM cells treated with $\mathrm{PZH}$ was determined by western blotting. $\beta$-actin was used as the internal control. Results are expressed as means \pm SD (error bars). Three individual experiments were performed. PZH, Pien Tze Huang; P-gp, P-glycoprotein.

cell lines. The $\mathrm{IC}_{50}$ of U2OS/ADM cells was quite similar to the U2OS cells, indicating that PZH has no cross-resistance to adriamycin and other classic anticancer drugs. The observations of the morphological changes and Annexin V/PI double staining analysis indicated that the percentage of apoptotic cells increased in a dose-dependent manner following incubation with different concentrations of PZH for $48 \mathrm{~h}$. These results suggest that PZH may play an important role in the treatment of patients with drug-resistant OS.

Cell cycle control is one of the major regulatory mechanisms of cancer cell division and duplication. When suffering drug-toxicity, cancer cells may activate cell cycle checkpoints to block cell cycle progression, which enhances damage repair and leads to a resistance phenotype. Many anticancer agents have been reported to arrest the cell cycle at a specific checkpoint $(11,35,36)$. Therefore, inhibiting the specific checkpoint of the cell cycle is one of the key approaches for the development of anticancer agents. To determine whether PZH inhibited cell proliferation via cell cycle arrest, flow cytometric analyses of the cell cycle were performed. We found that the effect of 
$\mathrm{PZH}$ in resistant U2OS/ADM cells was associated with cell cycle arrest at the $\mathrm{G}_{2} / \mathrm{M}$ phase in a dose-dependent manner, indicating that PZH inhibited U2OS/ADM cell proliferation by blocking the $\mathrm{G}_{2}$ to $\mathrm{M}$ progression, which may partly explain its mechanisms of antitumor activity.

Apoptosis, a type of programmed cell death, is a major mechanism of cell death following many types of chemotherapeutic agents. It is also closely related to chemotherapeutic drug resistance. Apoptosis occurs via two main routes, including the extrinsic and the intrinsic pathways. The intrinsic pathway is the major route for chemotherapy-induced apoptosis, and perturbation of this pathway may lead to considerable alterations in the response to chemotherapy (37). It has been confirmed that overexpression of Bcl-2 protein is correlated with chemotherapy resistance, and lentivirus-mediated Bcl-2 knockdown was found to sensitize human drug-resistant OS MG63 cells to doxorubicin (26). Similarly, the correlation of Bax expression levels with response to chemotherapy has generated conflicting reports (25). Our previous studies found that the expression of survivin in U2OS/ADM cells was significantly higher than that in the parental cells (31). Therefore, the upregulation of $\mathrm{Bcl}-2$ and survivin and the downregulation of Bax are related to the reduction in sensitivity to apoptosis in cancer cells. In the present study, an decrease in Bcl-2 and survivin and an increase in Bax were observed in U2OS/ADM cells following treatment with $\mathrm{PZH}$, suggesting that $\mathrm{PZH}$ may induce U2OS/ADM cell apoptosis.

Recent studies have demonstrated that P-gp does not only function as an energy-dependent drug pump to reduce intracellular chemical concentrations, but also leads to drug-resistance through inhibiting the activation of caspase-3 and -8 (38). As P-gp plays a key role in inhibiting apoptosis in drug-resistant cancer cells, effective inhibition of P-gp may be critical for providing a targeted site for cancer treatment for patients with drug resistance. The present study showed that the treatment of U2OS/ADM cells with PZH led to a dose-dependent decrease in P-gp mRNA and protein, accompanied by elevated Rhodamine 123 accumulation, which may result in apoptosis and cytotoxic effects in U2OS/ADM cells.

In conclusion, our data for the first time demonstrate that PZH inhibits U2OS/ADM cell proliferation via cell cycle $\mathrm{G}_{2} / \mathrm{M}$ arrest and enhanced apoptosis via the downregulation of the expression of Bcl-2, survivin and P-gp and upregulation of Bax. These data suggest that PZH has potential as a therapeutic agent against multidrug-resistant osteosarcoma and warrants further in vivo investigation.

\section{Acknowledgements}

The present study was supported by grants from the National Natural Science Foundation of China (nos. 30901916 and 81373659).

\section{References}

1. Mirabello L, Troisi RJ and Savage SA: Osteosarcoma incidence and survival rates from 1973 to 2004: data from the Surveillance, Epidemiology, and End Results Program. Cancer 115: 1531-1543, 2009.

2. Ottaviani G and Jaffe N: The epidemiology of osteosarcoma. Cancer Treat Res 152: 3-13, 2009.
3. Jaffe N, Puri A and Gelderblom H: Osteosarcoma: evolution of treatment paradigms. Sarcoma 2013: 203531, 2013.

4. Rajani R and Gibbs CP: Treatment of bone tumors. Surg Pathol Clin 5: 301-318, 2012.

5. Schwartz CL, Gorlick R, Teot L, et al: Multiple drug resistance in osteogenic sarcoma: INT0133 from the Children's Oncology Group. J Clin Oncol 25: 2057-2062, 2007.

6. Chou AJ and Gorlick R: Chemotherapy resistance in osteosarcoma: current challenges and future directions. Expert Rev Anticancer Ther 6: 1075-1085, 2006.

7. Saha SK and Khuda-Bukhsh AR: Molecular approaches towards development of purified natural products and their structurally known derivatives as efficient anti-cancer drugs: current trends. Eur J Pharmacol 714: 239-248, 2013.

8. Newman DJ and Cragg GM: Natural products as sources of new drugs over the 30 years from 1981 to 2010. J Nat Prod 75: 311-335, 2012.

9. Meng QX, Roubin RH and Hanrahan JR: Ethnopharmacological and bioactivity guided investigation of five TCM anticancer herbs. J Ethnopharmacol 148: 229-238, 2013.

10. Jia L, Ma S, Hou X, et al: The synergistic effects of traditional Chinese herbs and radiotherapy for cancer treatment. Oncol Lett 5: 1439-1447, 2013.

11. Wu G, Chu J, Huang Z, et al: Xiao Jin Wan, a traditional Chinese herbal formula, inhibits proliferation via arresting cell cycle progression at the $\mathrm{G} 2 / \mathrm{M}$ phase and promoting apoptosis via activating the mitochondrial-dependent pathway in U-2OS human osteosarcoma cells. Int J Oncol 42: 1070-1080, 2013.

12. Deng S, Hu B, An HM, et al: Teng-Long-Bu-Zhong-Tang, a Chinese herbal formula, enhances anticancer effects of 5-fluorouracil in CT26 colon carcinoma. BMC Complement Altern Med 13: 128, 2013.

13. Xu M, Sheng LH,Zhu XH,Zeng SB and Zhang GJ: Reversal effect of Stephania tetrandra-containing Chinese herb formula SENL on multidrug resistance in lung cancer cell line SW1573/2R120. Am J Chin Med 38: 401-413, 2010.

14. Yang L, Wei DD, Chen Z, Wang JS and Kong LY: Reversal effects of traditional Chinese herbs on multidrug resistance in cancer cells. Nat Prod Res 25: 1885-1889, 2011.

15. Chinese Pharmacopoeia Commission: Pharmacopoeia of the Peoples Republic of China. Chinese Med Sci Technol Press 1: 573-575, 2010.

16. Zhao SL and Pan J: A clinical trial of combined use of Pien Tze Huang and chemotherapy in the treatment of primary liver cancer. Med World 9: 40-51, 2006.

17. Xu YY and Yu EX: Clinical analysis of the effect of Pien Tze Huang in treatment of 42 patients with moderate or advanced liver cancer. Shanghai J Tradit Chin Med 12: 4-5, 1994.

18. Zhuang Q, Hong F, Shen A, et al: Pien Tze Huang inhibits tumor cell proliferation and promotes apoptosis via suppressing the STAT3 pathway in a colorectal cancer mouse model. Int J Oncol 40: 1569-1574, 2012.

19. Shen AL, Hong F, Liu LY, Lin JM, Zhuang QC, Hong ZF and Peng J: Effects of Pien Tze Huang on angiogenesis in vivo and in vitro. Chin J Integr Med 18: 431-436, 2012.

20. Zhang L, Yu B and Lin JH: Apoptosis induction of traditional Chinese herb Pianzihuang in human osteosarcoma U-2OS cells. Zhongguo Gu Shang 22: 265-268, 2009 (In Chinese).

21. Zhang L, Zeng QQ and Lin JH: Inhibitory effect of human P27KIP1 gene AVV virus combining with Chinese herb Pien Tze Huang on human osteosarcoma transplant mice model. China J TCM Pharm 24: 511-514, 2009.

22. Zhang Y, Wang QH, Zhang L, Niu SS and Liu Y: Effects of Pien Tze Huang on the cell cycle of human osteocarcom U2OS cells. J Jiangxi Univ TCM 24: 19-21, 2012.

23. Fu Y, Yang DH and Zhang L: Effects of Pien Tze Huang on the migration and invasion of osteosarcoma MG63 cell. China J TCM Pharm 28: 1577-1580, 2013.

24. Wu X, Cai ZD, Lou LM and Zhu YB: Expressions of p53, c-myc, $\mathrm{Bcl}-2$ and apoptotic index in human osteosarcoma and their correlations with prognosis of patients. Cancer Epidemiol 36: 212-216, 2012.

25. Wesarg E, Hoffarth S, Wiewrodt R, Kröll M, Biesterfeld S, Huber C and Schuler M: Targeting BCL-2 family proteins to overcome drug resistance in non-small cell lung cancer. Int J Cancer 121: 2387-2394, 2007.

26. Zhao Y, Zhang GL, Zeng BF, Wu XS, Gao TT and Oda Y: Enhanced chemosensitivity of drug-resistant osteosarcoma cells by lentivirus-mediated $\mathrm{Bcl}-2$ silencing. Biochem Biophys Res Commun 390: 642-647, 2009. 
27. Shoeneman JK, Ehrhart EJ III, Eickhoff JC, Charles JB, Powers BE and Thamm DH: Expression and function of survivin in canine osteosarcoma. Cancer Res 72: 249-259, 2012.

28. Serra M, Scotlandi K, Reverter-Branchat G, et al: Value of $\mathrm{P}$-glycoprotein and clinicopathologic factors as the basis for new treatment strategies in high-grade osteosarcoma of the extremities. J Clin Oncol 21: 536-542, 2003.

29. Gomes CM, van Paassen H, Romeo S, et al: Multidrug resistance mediated by $\mathrm{ABC}$ transporters in osteosarcoma cell lines: mRNA analysis and functional radiotracer studies. Nucl Med Biol 33: 831-840, 2006.

30. Johnstone RW, Ruefli AA, Tainton KM and Smyth MJ: A role for P-glycoprotein in regulating cell death. Leuk Lymphoma 38: $1-11,2000$.

31. Zhang Y, Liu JN, Wang QH, Liu Y and Zhang L: Comparison of characteristics of two adriamycin resistant human osteosarcoma U2OS cell lines. Acta Acad Med Weifang 34: 336-339, 2012.

32. Shen M, Chan TH and Dou QP: Targeting tumor ubiquitinproteasome pathway with polyphenols for chemosensitization. Anticancer Agents Med Chem 12: 891-901, 2012.
33. Milane L, Ganesh S, Shah S, Duan ZF and Amiji M: Multi-modal strategies for overcoming tumor drug resistance: hypoxia, the Warburg effect, stem cells, and multifunctional nanotechnology. J Control Release 155: 237-247, 2011.

34. Xu Y and Li XJ: Multi-target therapeutics and new drug discovery. Yao Xue Xue Bao 44: 226-230, 2009 (In Chinese).

35. Ji T, Lin C, Krill LS, Eskander R, Guo Y, Zi X and Hoang BH: Flavokawain B, a kava chalcone, inhibits growth of human osteosarcoma cells through $\mathrm{G}_{2} / \mathrm{M}$ cell cycle arrest and apoptosis. Mol Cancer 12: 55, 2013.

36. Zheng SE, Xiong S, Lin F, et al: Pirarubicin inhibits multidrugresistant osteosarcoma cell proliferation through induction of G2/M phase cell cycle arrest. Acta Pharmacol Sin 33: 832-838, 2012.

37. Mellor HR and Callaghan R: Resistance to chemotherapy in cancer: a complex and integrated cellular response. Pharmacology 81: 275-300, 2008.

38. Friedrich K, Wieder T, Von Haefen C, et al: Overexpression of caspase- 3 restores sensitivity for drug-induced apoptosis in breast cancer cell lines with acquired drug resistance. Oncogene 20: 2749-2760, 2001 